\title{
ANALYTIC SOLUTIONS FOR SOIL-STRUCTURE INTERACTION IN LAYERED MEDIA
}

\author{
GIN-SHOW LIOU* \\ Department of Civil Engineering, National Chiao-Tung University, Hsin-Chu, Taiwan
}

\begin{abstract}
SUMMARY
The total system studied in this paper is a layered soil stratum with a rigid bedrock and a cylindrical cavity on the surface. Analytic solutions for the layered medium with prescribed harmonic displacement time history on the surface of the cylindrical cavity are presented. The whole soil domain is divided into interior and exterior domains. The interior domain is the projection of the cylindrical cavity down to the rigid bedrock, whereas the exterior domain is then the soil medium complement to the interior domain. The displacement and stress fields in both domains are expanded as an infinite series of Fourier components with respect to the azimuth. For each Fourier component in the infinite series, the solutions for both domains are found independently by solving the general differential equations of wave propagation satisfying the boundary conditions of the top surface and the lower rigid boundary. Displacement and stress continuity conditions are then imposed on the vertical interface between the two domains using the formulation of a weighted residual. For the soil-structure interaction problem, the impedance matrix at the interface between the structure and the soil medium can be easily generated using the analytic solutions, which can then be combined with the finite element model of the structure. A simple example is presented to demonstrate the effectiveness of the procedure presented.
\end{abstract}

\section{INTRODUCTION}

The substructure technique is often used in seismic analyses involving soil-structure interactions. In such an application, the surrounding soil medium is represented by an impedance matrix, which can be easily combined into the total structural stiffness matrix, at the contact interface with the structure. Many different methods with different specific assumptions can be used to calculate the impedance matrix. In most cases, it is necessary to rely upon the finite element approach to model the soil in the vicinity of the soil-structure interface. $^{1,2}$ However, the finite element approach is expensive and sometimes ineffective, especially for general three dimensional cases. Therefore, much effort has been devoted to minimizing the finite element soil region.

To a certain extent, the boundary element method can serve the purpose of replacing the finite element approach in the soil domain. ${ }^{3,4}$ The conventional boundary element method uses the formulation of a weighted residual to minimize the error caused by the discrepancy between the Green's function and the finite element solution at the interface. ${ }^{5}$ To use the Green's function, a singularity problem will arise when the source point coincides with the observation point. To avoid this situation, one can choose a non-coincident observation point or use an analytic integration scheme. However, the complexity in the computation still exists for the general 3-D case. The analytic solutions presented in this paper can be employed in the boundary element method without the use of Green's functions.

Waas $^{6}$ and $\mathrm{Kausel}^{7}$ developed a semi-discrete analytic method to model the far-field with homogeneous boundary conditions for two dimensional and axisymmetric problems. This semi-discrete analytic model is then combined with the finite element model of the near-field to solve the soil-structure interaction problems in layered media. Tassoulas ${ }^{8}$ extended this method to calculate the semi-discrete analytic solutions for the

\footnotetext{
* Associate Professor, former Assistant Research Engineer, University of California, Berkeley.
} 
near-field with rigid foundation. Tzong and Penzien ${ }^{9}$ developed a boundary solution method to solve a similar problem. The major difference between the two methods is that Tzong's method uses a linear combination of the analytic solutions for the far-field while Waas' method discretizes the far-field by layer elements. Therefore, Tzong's method requires complex root finding of transcendental equations, while Waas' method solves an eigenvalue problem.

The research reported herein deals with the problem of soil-structure interaction in a layered medium having a rigid lower boundary. The total system shown in Figure 1 is divided into three domains: exterior and interior soil domains, and structural domain. Owing to the nature of the boundary conditions, the analytic solution for the interior domain is the combination of a homogeneous solution and a particular solution. The exterior domain is described by a homogeneous solution only. The homogeneous solution satisfies the boundary conditions of the rigid lower boundary and no prescribed excitation on the top surface, while the particular solution is directly determined from the prescribed excitation at the top surface of the interior domain.

Assuming harmonic displacement (or stress) time history is prescribed on the surface of the cylindrical cavity, the analytic solutions for both the exterior and the interior soil domains are generated independently. Then, displacement and stress continuities are imposed on the vertical interface between the exterior and the interior domains to obtain the participation factors of the homogeneous solutions. For the soil-structure interaction problem, the analytic solutions can be used to generate the impedance matrix at the surface of the cylindrical cavity by employing the formulation of a weighted residual to minimize the discrepancy between the analytic solution of the soil domain and the finite element solution of the structure.

To obtain the homogeneous solutions, one has to find the complex roots of the transcendental equations due to the homogeneous boundary conditions. A newly developed complex root searching scheme is proposed.

A simple example of a rigid massless circular plate resting on a single layer stratum and subjected to torsional and vertical motions is used to illustrate the procedure presented.

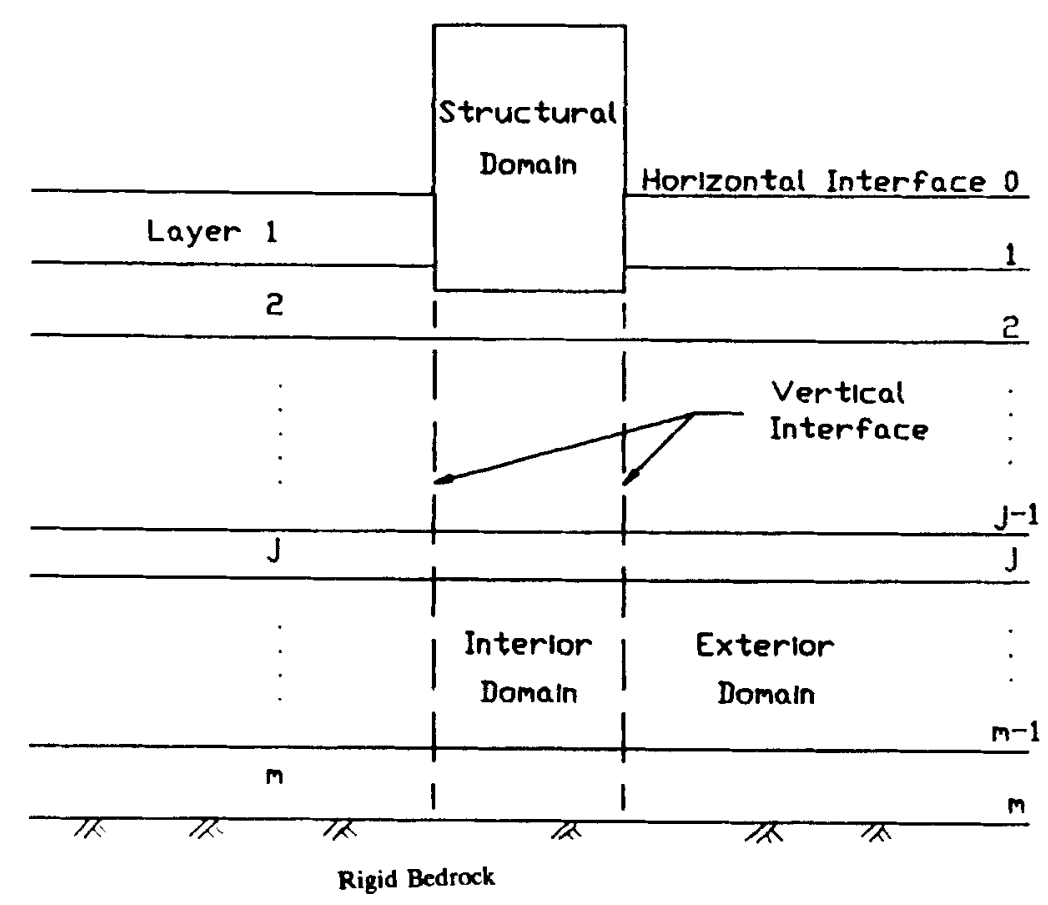

Figure 1. Total system 


\section{ANALYTIC SOLUTIONS FOR LAYERED MEDIA WITH CYLINDRICAL CAVITY}

The total system is shown in Figure 1. Let $u_{b 1}$ and $u_{b 2}$ in cylindrical coordinates be the harmonic displacement excitations on the wall and the bottom of the cylindrical cavity respectively (Figure 2).* One can express them in terms of Fourier components with respect to the azimuth as follows:

$$
u_{b 1}(\theta, z)=\sum_{n=0}^{n=\infty} F(\theta) \bar{u}_{b 1}^{n}(z)=\sum_{n=0}^{n=\infty}\left[\begin{array}{c}
\bar{u}_{r 1}^{n}(z)\left\{\begin{array}{l}
\cos n \theta \\
\sin n \theta
\end{array}\right. \\
\bar{u}_{z 1}^{n}(z)\left\{\begin{array}{c}
\cos n \theta \\
\sin n \theta
\end{array}\right. \\
\bar{u}_{\theta 1}^{n}(z)\left\{\begin{array}{c}
-\sin n \theta \\
\cos n \theta
\end{array}\right] r=a_{0} \quad \text { and } \quad 0 \leqslant z \leqslant d
\end{array}\right]
$$

and

$$
u_{b 2}(\theta, r)=\sum_{n=0}^{n=\infty} F(\theta) \bar{u}_{b 2}^{n}(r)=\sum_{n=0}^{n=\infty}\left[\begin{array}{c}
\bar{u}_{r 2}^{n}(r)\left\{\begin{array}{c}
\cos n \theta \\
\sin n \theta
\end{array}\right. \\
\bar{u}_{z 2}^{n}(r)\left\{\begin{array}{c}
\cos n \theta \\
\sin n \theta
\end{array}\right. \\
\bar{u}_{\theta 2}^{n}(r)\left\{\begin{array}{c}
-\sin n \theta \\
\cos n \theta
\end{array}\right] \quad 0 \leqslant r \leqslant a_{0} \quad \text { and } \quad z=d
\end{array}\right]
$$

where $\bar{u}_{r}^{n}, \bar{u}_{z}^{n}$ and $\bar{u}_{\theta}^{n}$ are the displacement amplitudes of the $n$th Fourier component in the $r, z$ and $\theta$ directions respectively. From superposition, it is sufficient to describe the solution procedure by considering only a particular Fourier displacement component in equations (1) and (2).

The general equations of wave propagation with harmonic excitations can be expressed in cylindrical coordinates as follows:

$$
\begin{aligned}
& -\omega^{2} \rho u_{r}=(\lambda+2 G) \frac{\partial \Delta}{\partial r}-\frac{2 G}{r} \frac{\partial w_{r}}{\partial \theta}+2 G \frac{\partial w_{\theta}}{\partial z} \\
& -\omega^{2} \rho u_{z}=(\lambda+2 G) \frac{\partial \Delta}{\partial z}-\frac{2 G}{r} \frac{\partial\left(r w_{\theta}\right)}{\partial r}+\frac{2 G}{r} \frac{\partial w_{r}}{\partial \theta} \\
& -\omega^{2} \rho u_{\theta}=\frac{(\lambda+2 G)}{r} \frac{\partial \Delta}{\partial \theta}-2 G \frac{\partial w_{r}}{\partial z}+2 G \frac{\partial w_{z}}{\partial r}
\end{aligned}
$$

where $\lambda$ and $G$ are Lamé's constants, $\rho$ is the mass density, $\omega$ is the frequency,

$$
\Delta=\frac{1}{r} \frac{\partial\left(r u_{r}\right)}{\partial r}+\frac{1}{r} \frac{\partial u_{\theta}}{\partial \theta}+\frac{\partial u_{z}}{\partial z}
$$

is the dilatation, and

and

$$
2 w_{r}=\frac{1}{r} \frac{\partial u_{z}}{\partial \theta}-\frac{\partial u_{\theta}}{\partial z}, \quad 2 w_{z}=\frac{1}{r}\left(\frac{\partial\left(r u_{\theta}\right)}{\partial r}-\frac{\partial u_{r}}{\partial \theta}\right)
$$

$$
2 w_{\theta}=\frac{\partial u_{r}}{\partial z}-\frac{\partial u_{z}}{\partial r}
$$

are the rotations.

* For convenience, the harmonic time variation $\mathrm{e}^{\mathrm{i} \omega t}$ has been omitted in the following derivation. 


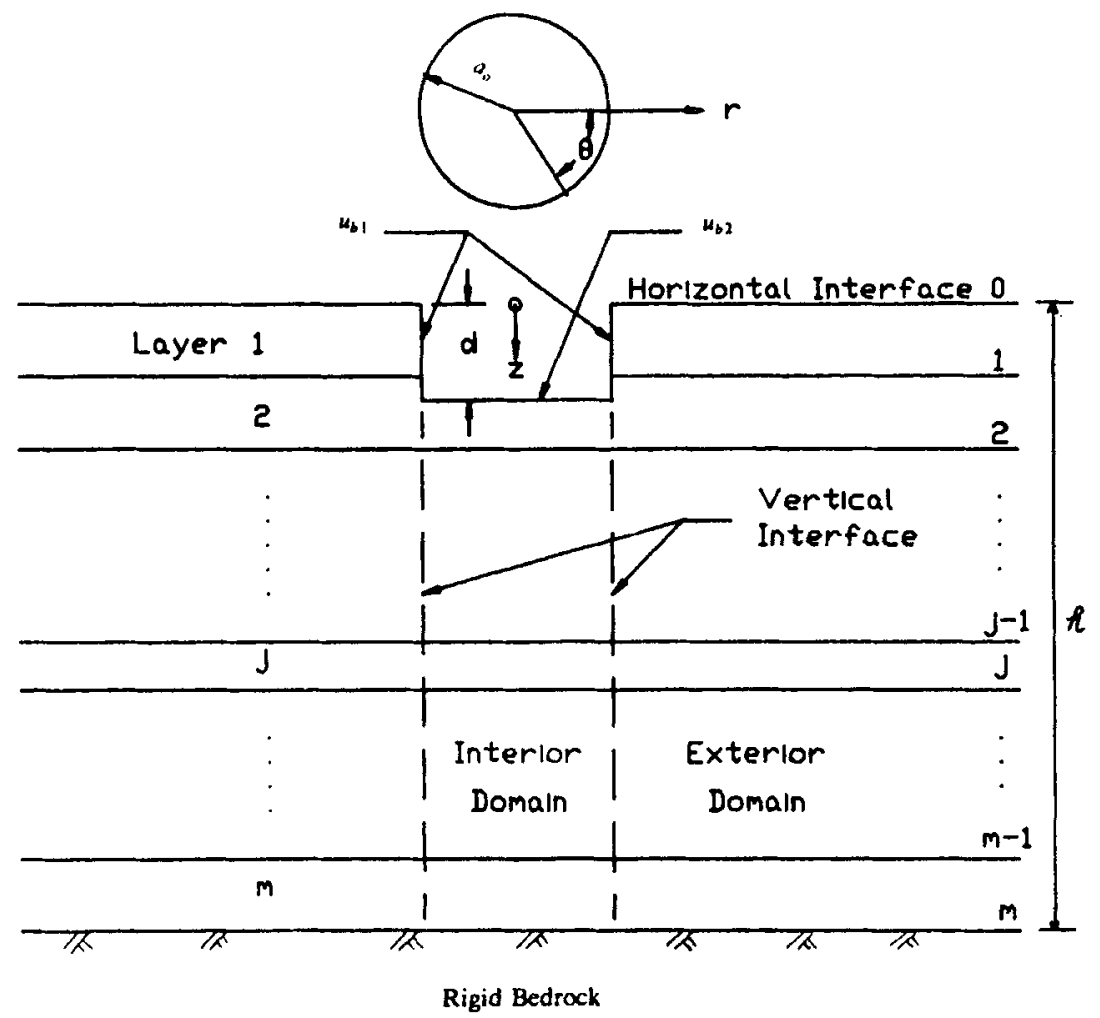

Figure 2. Layered soil domain

Referring to Figure 2, the solutions of equation (3) for the $n$th Fourier component must satisfy the following boundary conditions:

1. Free surface condition: the tractions at $z=0$ and $r \geqslant a_{0}$ must be equal to zero.

2. Rigid base condition: the displacements at $z=h$ must be equal to zero.

3. Radiation condition: both displacements and stresses must be equal to zero when $r \rightarrow \infty$.

4. Displacement boundary on the wall of the cavity: the displacements at $r=a_{0}$ and $0 \leqslant z \leqslant d$ must be equal to $\bar{u}_{b 1}^{n}$ in equation (1).

5. Displacement boundary on the bottom of the cavity: the displacements at $z=d$ and $0 \leqslant r \leqslant a_{0}$ must be equal to $\bar{u}_{b 2}^{n}$ in equation (2).

For convenience, the superscript $n$ in equations (1) and (2) and in the boundary conditions (4) and (5) are dropped in the formulations that follow.

To solve equation (3) satisfying the boundary conditions (1)-(5), one can divide the soil domain into the exterior and the interior domains as shown in Figure 2. The solution $u^{(e)}=\left(u_{r}^{(e)}, u_{z}^{(e)}, u_{\theta}^{(e)}\right)^{\mathrm{T}}$ of equation (3) for the exterior domain now should satisfy the boundary conditions (1), (2), (3) and (4) and the condition of displacement continuity at the vertical interface with the interior domain. The condition (4) and the condition of displacement continuity can be written as

$$
u^{(\mathrm{e})}= \begin{cases}\bar{u}_{b 1} & r=a_{0} \text { and } 0 \leqslant z \leqslant d \\ u^{(\mathrm{i})} & r=a_{0} \text { and } d \leqslant z \leqslant h\end{cases}
$$

The solution $u^{(\mathrm{i})}=\left(u_{r}^{(\mathrm{i})}, u_{z}^{(\mathrm{i})}, u_{\theta}^{(\mathrm{i})}\right)^{\mathrm{T}}$ of equation (3) for the interior domain should satisfy the boundary conditions (2) and (5) and the condition of stress continuity at the vertical interface. The condition of stress continuity can 
be written as

$$
t^{(\mathbf{i})}=t^{(\mathrm{e})} \quad r=a_{0} \quad \text { and } \quad d \leqslant z \leqslant h
$$

where $t^{(\mathrm{i})}=\left(\sigma_{r r}^{(\mathrm{i})}, \tau_{r z}^{(\mathrm{i})}, \tau_{r \theta}^{(\mathrm{i})}\right)^{\mathrm{T}}$ and $t^{(\mathrm{e})}=\left(\sigma_{r r}^{(\mathrm{e})}, \tau_{r z}^{(\mathrm{e})}, \tau_{r \theta}^{(\mathrm{e})}\right)^{\mathrm{T}}$.

\subsection{Solutions for exterior domain}

The exterior domain is shown in Figure 3(a). For the $n$th Fourier component, Sezawa ${ }^{10}$ has developed a procedure to separate the dilatational and the rotational waves in equation (3) and used the technique of the separation of variables to obtain the general solution of equation (3) for the half-space medium. Tzong and Penzien ${ }^{9}$ extended this solution to the layered medium and described the stress and the displacement fields in a layer in terms of the displacements and the tractions on the upper boundary of the layer. By imposing the displacement and the stress continuities between two layers consecutively from the top layer to the bottom layer, one obtains

$$
Y_{m}^{(\mathrm{e})}=\mathbf{H a} \mathbf{a}_{m-1} \mathrm{a}_{m-1} \cdots \mathbf{a}_{1} \mathbf{H}^{-1} Y_{0}^{(\mathrm{e})}=\mathbf{H} \mathbf{T}^{(\mathrm{e})} \mathbf{H}^{-1} Y_{0}^{(\mathrm{e})}
$$

where $Y_{m}^{(\mathrm{e})}=\left(\bar{u}_{r}^{(\mathrm{e})}, \bar{u}_{z}^{(\mathrm{e})}, \bar{\tau}_{r z}^{(\mathrm{e})}, \bar{\sigma}_{z z}^{(\mathrm{e})}, \bar{u}_{\theta}^{(\mathrm{e})}, \bar{\tau}_{\theta z}^{(\mathrm{e})}\right)_{m}^{\mathrm{T}}$ is the displacement-stress vector on the $m$ th horizontal interface in Figure 3(a), $\mathbf{H}$ is the Hankel function matrix and the $\mathbf{a}_{j}$ 's are the transfer matrices given by equations (A4),

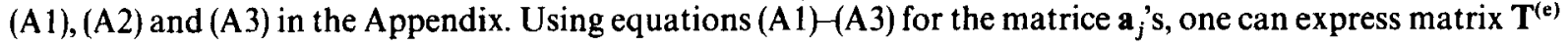
in the following form:

$$
\mathbf{T}^{(\mathrm{e})}=\left[\begin{array}{cccccc}
t_{11} & t_{12} & t_{13} & t_{14} & 0 & 0 \\
t_{21} & t_{22} & t_{23} & t_{24} & 0 & 0 \\
t_{31} & t_{32} & t_{33} & t_{34} & 0 & 0 \\
t_{41} & t_{42} & t_{43} & t_{44} & 0 & 0 \\
0 & 0 & 0 & 0 & t_{55} & t_{56} \\
0 & 0 & 0 & 0 & t_{65} & t_{66}
\end{array}\right]
$$

From the bounndary conditions of the free surface and the rigid lower boundary, equation (6) gives the transcendental equations

$$
t_{11} t_{22}-t_{12} t_{21}=0
$$

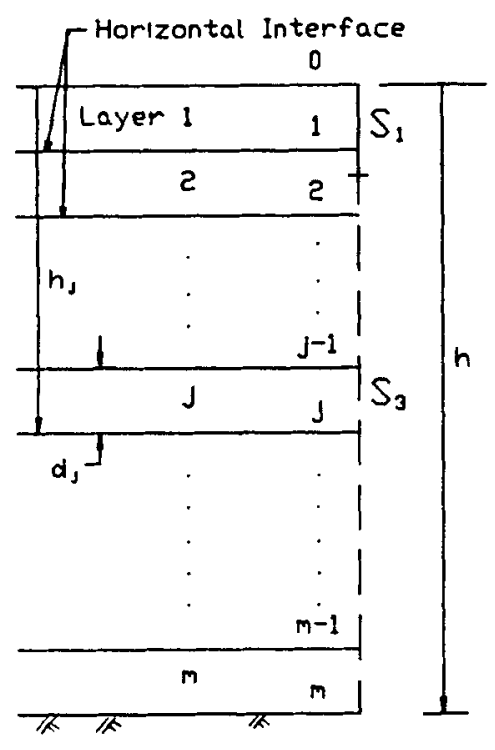

Figure 3(a). Exterior domain 
for the wave numbers representing Rayleigh modes, and

$$
t_{55}=0
$$

for the wave numbers representing Love modes. For each wave number $k$ which is a root of equation (8) or (9), the tractions at depth $z$ on the vertical interface between the exterior and the interior domains can be expressed in terms of the displacement-stress vector on the free surface as follows:

$$
t_{j}^{(\mathrm{e})}(z)=\left(\mathbf{H}_{1} \mathbf{F}_{1}+\mathbf{H}_{2} \mathbf{F}_{2}\right) e\left(z-h_{j-1}\right) \mathbf{E}_{j}^{-1} \mathbf{a}_{j-1} \ldots \mathbf{a}_{1} \mathbf{H}^{-1} Y_{0}^{(\mathbf{e})}
$$

where $t_{j}^{(\mathrm{e})}(z)=\left.\left(\sigma_{r r}^{(\mathrm{e})}, \tau_{r z}^{(\mathrm{e})}, \tau_{r \theta}^{(\mathrm{e})}\right)^{\mathrm{T}}\right|_{r=a_{0}}$ in the $j$ th layer, and the matrices $\mathbf{H}_{1}, \mathbf{H}_{2}, \mathbf{F}_{1} e\left(z-h_{j-1}\right) \mathbf{E}_{j}^{-1}$ and $\mathbf{F}_{2} e\left(z-h_{j-1}\right)$ $\mathbf{E}_{j}^{-1}$ are given by equations (A5)-(A8) in the Appendix.

Substituting the root of equation (8) into equation (6) and making use of the free surface and the lower rigid boundary conditions, one can easily show that $\mathbf{H}^{-1} Y_{0}^{(\mathrm{e})}$ in equations (6) and (10) can be written as

$$
\mathbf{H}^{-1} Y_{0}^{(\mathrm{e})}=\left(1, \xi_{i}, 0,0,0,0\right)^{\mathrm{T}} \alpha_{i}^{(\mathrm{e})}
$$

for the $i$ th Rayleigh mode, in which $\xi_{i}=-t_{11} / t_{12}=-t_{21} / t_{22}$ and $\alpha_{i}^{(\mathrm{e})}$ is the unknown modal participation factor. Similarly, substituting the root of equation (9) into equation (6), one obtains

$$
\mathbf{H}^{-1} Y_{0}^{(\mathrm{e})}=(0,0,0,0,1,0)^{\mathrm{T}} \alpha_{j}^{(e)}
$$

for the $j$ th Love mode, in which $\alpha_{j}^{(e)}$ is the unknown modal participation factor. Although equations (8) and (9) have an infinite number of roots, the displacement and the stress fields in the exterior domain can be approximated adequately by only a few lower modes. The number of modes needed depends upon the desired accuracy.

After the number of modes to approximate the displacement and the stress fields in the exterior domain is determined, the displacement and the stress vectors on the vertical interface (vertical surface $S_{1}+S_{3}$ in Figure 3(a)) can be expressed by the combination of these modes with unknown participation factors as follows:

$$
\begin{aligned}
u^{(e)}(z) & =\mathbf{N}^{(\mathrm{e})}(z) \alpha^{(\mathrm{e})} \\
t^{(\mathrm{e})}(z) & =\mathbf{G}^{(\mathrm{e})}(z) \alpha^{(\mathrm{e})}
\end{aligned}
$$

where $\mathbf{N}^{(e)}(z)$ and $\mathbf{G}^{(e)}(z)$ are the matrices of modal displacements and stresses respectively, and $\alpha^{(e)}$ is the vector of unknown modal participation factors in equations (11) and (12).

\subsection{Solutions for interior domain}

The interior domain is shown in Figure 3(b). Since the solution for the interior domain has to satisfy the non-homogeneous boundary condition (5), the solution is the combination of the homogeneous and the particular solutions: i.e.

$$
u^{(i)}=u_{\mathrm{h}}^{(\mathrm{i})}+u_{\mathrm{p}}^{(\mathrm{i})}
$$

The particular solution $u_{\mathrm{p}}^{(\mathrm{i})}$ satisfies the boundary conditions (2) and (5), whereas the homogeneous solution $u_{\mathrm{h}}^{(\mathrm{i})}$ satisfies the boundary condition (2) and

$$
\left.u_{\mathrm{h}}^{(\mathrm{i})}\right|_{z=\mathrm{d}}=0 \quad 0 \leqslant r \leqslant a_{0}
$$

2.2.1. Homogeneous solution. To obtain the homogeneous solution, one can follow the procedure described in the preceding section. As explained for equation (6), a similar equation can be obtained by applying the displacement and the stress continuities between two layers consecutively from the top layer to the bottom layer. The equation is

$$
Y_{n}^{(i)}=\mathbf{J a}_{n} \mathbf{a}_{n-1} \ldots \mathbf{a}_{1} \mathbf{J}^{-1} Y_{0}^{(i)}=\mathbf{J T}^{(i)} \mathbf{J}^{-1} Y_{0}^{(i)}
$$

where $Y_{n}^{(\mathrm{i})}=\left(\bar{u}_{r}^{(\mathrm{i})}, \bar{u}_{z}^{(\mathrm{i})}, \bar{\tau}_{r z}^{(\mathrm{i})}, \bar{\sigma}_{z z}^{(\mathbf{i})}, \bar{u}_{\theta}^{(\mathrm{i})}, \bar{\tau}_{\theta z}^{(\mathrm{i})}\right)_{n}^{\mathbf{T}}$ is the displacement-stress vector on the $n$th horizontal interface in Figure 3(b), matrix $T^{(i)}$ is similar to $T^{(\mathbf{e})}$ in equation (7) and matrix $J$ is similar to $\mathbf{H}$ in equation (A4) in the 


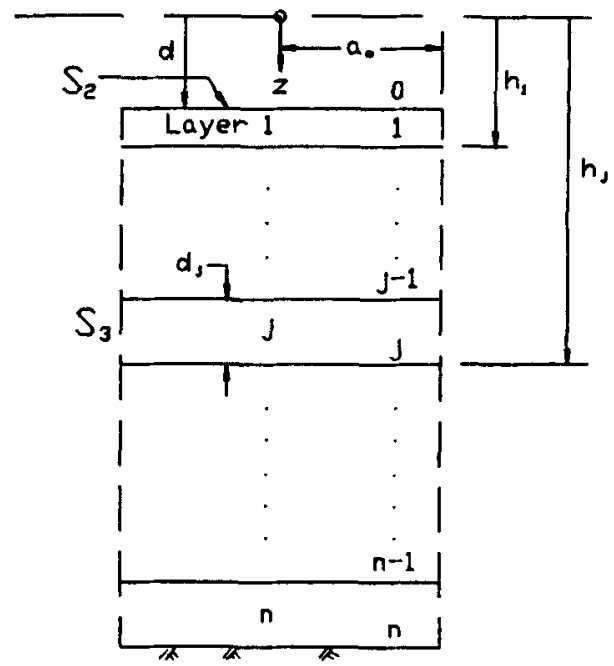

Figure 3(b). Interior domain

Appendix with the Hankel function in $\mathbf{H}$ replaced by the Bessel function. Because of equation (15) and the rigid lower boundary, equation (16) gives the following transcendental equations:

$$
t_{13}^{(i)} t_{24}^{(i)}-t_{14}^{(i)} t_{23}^{(i)}=0
$$

for the wave numbers representing Rayleigh modes, and

$$
t_{56}^{(i)}=0
$$

for the wave numbers representing Love modes, where $t_{i j}^{(\mathbf{i})}$ is the element $i j$ in the matrix $\mathbf{T}^{(\mathbf{i})}$. Similarly, for each root of equations (17) and (18), the traction $t_{j}^{(i)}(z)$ at depth $z$ on the vertical interface can be expressed as

$$
t_{j}^{(\mathrm{i})}(z)=\left(\mathbf{J}_{1} \mathbf{F}_{1}+\mathbf{J}_{2} \mathbf{F}_{2}\right) e\left(z-h_{j-1}\right) \mathbf{E}_{j}^{-1} \mathbf{a}_{j-1} \ldots \mathbf{a}_{1} \mathbf{J}^{-1} Y_{0}^{(\mathrm{i})}
$$

where the matrices $\mathbf{J}_{1}, \mathbf{J}_{2}$ are similar to $\mathbf{H}_{1}, \mathbf{H}_{2}$ in equations (A7) and (A8) in the Appendix with the Hankel function in $\mathbf{H}_{1}$ and $\mathbf{H}_{2}$ replaced by the Bessel function, and $\mathbf{F}_{1} e\left(z-h_{j-1}\right) E_{j}^{-1}$ and $F_{2} e\left(z-h_{j-1}\right) E_{j}^{-1}$ are shown in equation (A5) and (A6) in the Appendix. As explained in the derivations of equations (11) and (12), one can substitute the conditions of equation (15) and the rigid lower boundary into equation (16) to obtain $\mathbf{J}^{-1} Y_{0}^{(i)}$ for each root of equations (17) and (18). Therefore, $\mathbf{J}^{-1} Y_{0}^{(i)}$ in equations (16) and (19) is

$$
\mathbf{J}^{-1} Y_{0}^{(\mathbf{i})}=\left(0,0,1, \eta_{i}, 0,0\right)^{\mathrm{T}} \alpha_{i}^{(\mathbf{i})}
$$

and

$$
\mathbf{J}^{-1} Y_{0}^{(\mathrm{i})}=(0,0,0,0,0,1)^{\mathrm{r}} \alpha_{j}^{(\mathrm{i})}
$$

for the $i$ th Rayleigh mode and the $j$ th Love mode respectively, where $\alpha_{i}^{(i)}$ and $\alpha_{j}^{(i)}$ are the unknown modal participation factors, and

$$
\eta_{i}=-\frac{t_{13}^{(i)}}{t_{14}^{(i)}}=-\frac{t_{23}^{(i)}}{t_{24}^{(i)}}
$$

Similar to the solution for the exterior domain, the displacement and stress vectors on the upper boundary and the vertical interface, $S_{2}$ and $S_{3}$ in Figure 3(b), can be approximated by a finite number of lower modes such that

$$
\begin{aligned}
& u_{\mathrm{h}, s 2}^{(\mathrm{i})}(r)=0 \\
& t_{\mathrm{h}, s \mathbf{2}}^{(\mathbf{i})}(r)=\mathbf{G}_{\mathrm{h}, \mathbf{s} 2}^{(\mathbf{i})}(r) \alpha^{(\mathbf{i})}
\end{aligned}
$$


and

$$
\begin{aligned}
u_{\mathrm{h}, s 3}^{(\mathrm{i})}(z) & =\mathbf{N}_{\mathrm{h}, \mathrm{s3}}^{(\mathrm{i})}(z) \alpha^{(\mathrm{i})} \\
t_{\mathrm{h}, s 3}^{(\mathrm{i})}(z) & =\mathbf{G}_{\mathrm{h}, \mathbf{s} 3}^{(\mathrm{i})}(z) \alpha^{(\mathrm{i})}
\end{aligned}
$$

2.2.2. Particular solution. Since the particular solution must satisfy the non-homogeneous boundary condition (5), a special technique is developed to impose the non-homogeneous boundary condition on the general solution of equation (3) for the interior domain. Referring to Figure 3(b) and considering the first top layer, the general solution of equation (3) for the $n$th Fourier component in the interior domain can be obtained using the procedure reported by Sezawa ${ }^{10}$ to separate the dilatational and the rotational waves in equation (3), as briefly discussed in Section 2.1 . The general solution is

$$
\left\{\begin{array}{l}
u_{r}(r, z) \\
u_{z}(r, z) \\
u_{\theta}(r, z)
\end{array}\right\}=\left[\begin{array}{ccc}
J_{n}^{\prime}(k r) & 0 & \frac{n}{r} J_{n}(k r) \\
0 & k J_{n}(k r) & 0 \\
\frac{n}{r} J_{n}(k r) & 0 & J_{n}^{\prime}(k r)
\end{array}\right]\left[\begin{array}{cccccc}
k & k & 0 & 0 & -v^{\prime} & v^{\prime} \\
-v & v & 0 & 0 & k & k \\
0 & 0 & 1 & 1 & 0 & 0
\end{array}\right] e A
$$

or

$$
u=\mathbf{J}_{n} \mathbf{K} e A
$$

where $J_{n}^{\prime}(k r)=\mathrm{d}\left(J_{n}(k r)\right) / \mathrm{d} r, e=\operatorname{diag}\left(\mathrm{e}^{-v(z-d)}, \mathrm{e}^{v(z-d)}, \mathrm{e}^{-v^{\prime}(z-d)}, \mathrm{e}^{v^{\prime}(z-d)}, \mathrm{e}^{-v^{\prime}(z-d)}, \mathrm{e}^{\nu^{\prime}(z-d)}\right), v=\sqrt{k^{2}-\left(\omega^{2} / c_{\mathrm{p}}^{2}\right)}$, $v^{\prime}=\sqrt{k^{2}-\left(\omega^{2} / c_{\mathrm{s}}^{2}\right)}, A=\left(A_{1}, A_{2}, B_{1}, B_{2}, C_{1}, C_{2}\right)^{\mathrm{T}}$ are unknown coefficients determined from the boundary conditions, and $c_{\mathrm{p}}$ and $c_{\mathrm{s}}$ are the compressional and shear wave velocities respectively. One can obtain the displacements at the upper boundary by substituting $z=d$ into equation (24); i.e.

$$
\left.u\right|_{z=d}=\mathbf{J}_{n} \mathbf{K} A
$$

In the right-hand side, one can see that only $\mathbf{J}_{n}$ is a function of $r$ and can be decomposed, using the technique of expressing a matrix in terms of its eigenvectors and eigenvalues, as follows:

$$
\mathbf{J}_{n}=\left[\begin{array}{rrr}
1 & 0 & 1 \\
0 & 1 & 0 \\
-1 & 0 & 1
\end{array}\right]\left[\begin{array}{ccc}
-k J_{n+1}(k r) & 0 & 0 \\
0 & k J_{n}(k r) & 0 \\
0 & 0 & k J_{n-1}(k r)
\end{array}\right]\left[\begin{array}{rrr}
1 & 0 & 1 \\
0 & 1 & 0 \\
-1 & 0 & 1
\end{array}\right]^{-1}
$$

or

$$
\mathbf{J}_{n}=S \Lambda S^{-1}
$$

in which matrix $S$ is formed by the orthogonal eigenvectors of $J_{n}$ and $\Lambda$ is the diagonal eigenvalue matrix. Let $D=\mathbf{K} A=\left(d_{1}, d_{2}, d_{3}\right)^{\mathrm{T}}$ in equation (25) be expressed in terms of $S$; i.e.

$$
D=S\left\{\begin{array}{c}
\frac{d_{1}-d_{3}}{2} \\
d_{2} \\
\frac{d_{1}+d_{3}}{2}
\end{array}\right\}
$$

or

$$
D=S D^{\prime}
$$


Substituting equations (26) and (27) into equation (25), one obtains

$$
\left.u\right|_{z=d}=S \Lambda D^{\prime}
$$

Similarly, the prescribed boundary displacements $\bar{u}_{b 2}$ at the bottom of the cavity [boundary condition (5)] can also be written in terms of $S$; i.e.

$$
\left\{\begin{array}{l}
\bar{u}_{r 2}(r) \\
\bar{u}_{z 2}(r) \\
\bar{u}_{\theta 2}(r)
\end{array}\right\}=S\left\{\begin{array}{c}
\frac{\bar{u}_{r 2}(r)-\bar{u}_{\theta 2}(r)}{2} \\
\bar{u}_{z 2}(r) \\
\frac{\bar{u}_{r 2}(r)+\bar{u}_{\theta 2}(r)}{2}
\end{array}\right\}
$$

or

$$
\bar{u}_{b 2}=S \bar{u}_{b 2}^{\prime}
$$

Comparing equation (29) with equation (28) and enforcing the boundary condition (5), one can conclude that each element in $\bar{u}_{b 2}^{\prime}$ should be expanded as a series of Bessel functions in the interval $\left(0, a_{0}\right)$ in which $a_{0}$ is the radius of the cavity; i.e.

$$
\begin{aligned}
\frac{\bar{u}_{r 2}-\bar{u}_{\theta 2}}{2} & =\sum_{i=0}^{\infty}-k_{i}^{(1)} J_{n+1}\left(k_{i}^{(1)} r\right) \beta_{i}^{(1)} \\
\bar{u}_{z 2} & =\sum_{j=0}^{\infty} k_{j}^{(2)} J_{n}\left(k_{j}^{(2)} r\right) \beta_{j}^{(2)} \\
\frac{\bar{u}_{r 2}+\bar{u}_{\theta 2}}{2} & =\sum_{l=0}^{\infty} k_{l}^{(3)} J_{n-1}\left(k_{l}^{(3)} r\right) \beta_{l}^{(3)}
\end{aligned}
$$

where the $k_{i}^{(1) ' s,} k_{j}^{(2) ' s ~ a n d ~} k_{l}^{(3) ' s}$ are the roots of $J_{n+1}\left(k a_{0}\right)=0, J_{n}\left(k a_{0}\right)=0$, and $J_{n-1}\left(k a_{0}\right)=0$ respectively, for $i, j$, $l=1,2, \ldots, \infty$, and choosing $k_{0}^{(1)}=0.5 k_{1}^{(1)}, k_{0}^{(2)}=0.5 k_{1}^{(2)}$ and $k_{0}^{(3)}=0.5 k_{1}^{(3)}$ in order to satisfy the boundary condition at $r=a_{0}$ and $z=d .^{*}$ The Bessel functions in equations (30), (31) or (32), except the first term, are orthogonal to each other with respect to the weighting function $w(r)=r$ in the interval $\left(0, a_{0}\right)$. The $\beta_{i}^{(1)}$ 's, $\beta_{j}^{(2)}$ 's and $\beta_{l}^{(3)}$ 's can be determined from the orthogonal property as follows:

$$
\begin{gathered}
\beta_{0}^{(1)}=-\frac{\left.\left(\bar{u}_{r 2}-\bar{u}_{\theta 2}\right)\right|_{r=a_{0}}}{2 k_{0}^{(1)} J_{n+1}\left(k_{0}^{(1)} a_{0}\right)} \\
\beta_{i}^{(1)}=-\frac{\int_{0}^{a_{0}} r \frac{\tilde{u}_{r 2}-\bar{u}_{\theta 2}}{2} J_{n+1}\left(k_{i}^{(1)} r\right) \mathrm{d} r+\beta_{0}^{(1)} k_{0}^{(1)} \int_{0}^{a_{0}} r J_{n+1}\left(k_{0}^{(1)} r\right) J_{n+1}\left(k_{i}^{(1)} r\right) \mathrm{d} r}{k_{i}^{(1)} \int_{0}^{a_{0}} r J_{n+1}^{2}\left(k_{i}^{(1)} r\right) \mathrm{d} r} \quad i=1,2, \ldots, \infty \\
\beta_{0}^{(2)}=\frac{\left.\bar{u}_{22}\right|_{r=a_{0}}}{k_{0}^{(2)} J_{n}\left(k_{0}^{(2)} a_{0}\right)} \\
\beta_{j}^{(2)}=\frac{\int_{0}^{a_{0}} r \bar{u}_{z 2} J_{n}\left(k_{j}^{(2)} r\right) \mathrm{d} r-\beta_{0}^{(2)} k_{0}^{(2)} \int_{0}^{a_{0}} r J_{n}\left(k_{0}^{(2)} r\right) J_{n}\left(k_{j}^{(2)} r\right) \mathrm{d} r}{k_{j}^{(2)} \int_{0}^{a_{0}} r J_{n}^{2}\left(k_{j}^{(2)} r\right) \mathrm{d} r} j=1,2, \ldots, \infty
\end{gathered}
$$

* This condition may not be necessary to be satisfied. However, this treatment can reduce the number of terms needed to approximate the boundary condition. 


$$
\begin{gathered}
\beta_{0}^{(3)}=\frac{\left.\left(\bar{u}_{r 2}+\bar{u}_{\theta 2}\right)\right|_{r=a_{0}}}{2 k_{0}^{(3)} J_{n-1}\left(k_{0}^{(3)} a_{0}\right)} \\
\beta_{l}^{(3)}=\frac{\int_{0}^{a_{0}} r \frac{\bar{u}_{r 2}+\bar{u}_{\theta 2}}{2} J_{n-1}\left(k_{l}^{(3)} r\right) \mathrm{d} r-\beta_{0}^{(3)} k_{0}^{(3)} \int_{0}^{a_{0}} r J_{n-1}\left(k_{0}^{(3)} r\right) J_{n-1}\left(k_{l}^{(3)} r\right) \mathrm{d} r}{k_{l}^{(3)} \int_{0}^{a_{0}} r J_{n-1}^{2}\left(k_{l}^{(3)} r\right) \mathrm{d} r} l=1,2, \ldots, \infty
\end{gathered}
$$

The terms $-k_{i}^{(1)} J_{n+1}\left(k_{i}^{(1)} r\right), k_{j}^{(2)} J_{n}\left(k_{j}^{(2)} r\right)$ and $k_{l}^{(3)} J_{n-1}\left(k_{l}^{(3)} r\right)$ in equations (30)-(32) are the eigenvalues of the matrix $\mathbf{J}_{n}$ in equation (26). Therefore, the prescribed displacement $\bar{u}_{b 2}$ [equations (29)-(32)] can be expanded as

$$
\begin{aligned}
\left\{\begin{array}{l}
\bar{u}_{r 2}(r) \\
\bar{u}_{22}(r) \\
\bar{u}_{\theta 2}(r)
\end{array}\right\} & =\sum_{i=0}^{\infty}-\left\{\begin{array}{r}
1 \\
0 \\
-1
\end{array}\right\} k_{i}^{(1)} J_{n+1}\left(k_{i}^{(1)} r\right) \beta_{i}^{(1)}+\sum_{j=0}^{\infty}\left\{\begin{array}{l}
0 \\
1 \\
0
\end{array}\right\} k_{j}^{(2)} J_{n}\left(k_{j}^{(2)} r\right) \beta_{j}^{(2)}+\sum_{i=0}^{\infty}\left\{\begin{array}{l}
1 \\
0 \\
1
\end{array}\right\} k_{l}^{(3)} J_{n-1}\left(k_{l}^{(3)} r\right) \beta_{l}^{(3)} \\
& =\sum_{i=0}^{\infty} \mathbf{J}_{n}^{i}\left\{\begin{array}{r}
1 \\
0 \\
-1
\end{array}\right\} \beta_{i}^{(1)}+\sum_{j=0}^{\infty} \mathbf{J}_{n}^{j}\left\{\begin{array}{l}
0 \\
1 \\
0
\end{array}\right\} \beta_{j}^{(2)}+\sum_{i=0}^{\infty} \mathbf{J}_{n}^{l}\left\{\begin{array}{l}
1 \\
0 \\
1
\end{array}\right\} \beta_{l}^{(3)}
\end{aligned}
$$

where $\mathbf{J}_{n}^{i}, \mathbf{J}_{n}^{j}$ and $\mathbf{J}_{n}^{l}$ for mode $i, j$ and $l$ respectively are defined in equations (24) or (26). In equation (36), the $\beta_{i}^{(1)}$ 's, $\beta_{j}^{(2)}$ 's and $\beta_{l}^{(3)}$ 's are defined as the modal participation factors with respect to the wave number $k_{i}^{(1)}$ 's,

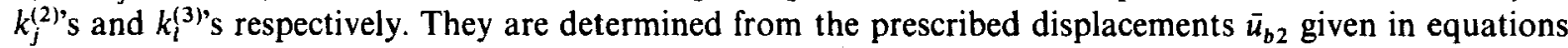
(33)-(35).

The preceding derivations have shown that the arbitary presribed displacements on the upper boundary can be decomposed into three separate series of Bessel function matrices [equation (36)]. Each mode in the series satisfies the general solution [equation (24)] of equation (3) on the upper boundary. Similar to the homogeneous solutions, the displacement and the stress fields given by the particular solutions can also be expressed in terms of the displacements and the stresses on the upper boundary of the interior domain as shown in equations (16) and (19). For each mode in the series shown in equation (36), one can apply the displacement and the stress continuities on the horizontal interfaces consecutively down to the lower rigid boundary as shown in equation (16). By making use of equation (16) and introducing the condition of rigid lower boundary, the corresponding traction vector on the upper boundary is then

$$
\mathbf{J}_{n}^{j-1} \bar{t}_{b 2}^{j}=-\left[\begin{array}{ccc}
t_{13}^{(\mathrm{i})} & t_{14}^{(\mathrm{i})} & 0 \\
t_{23}^{(\mathrm{i})} & t_{24}^{(\mathrm{i})} & 0 \\
0 & 0 & t_{56}^{(\mathrm{i})}
\end{array}\right]^{-1}\left[\begin{array}{ccc}
t_{11}^{(\mathrm{i})} & t_{12}^{(\mathrm{i})} & 0 \\
t_{21}^{(\mathrm{i})} & t_{22}^{(\mathrm{i})} & 0 \\
0 & 0 & t_{55}^{(\mathrm{i})}
\end{array}\right] \mathbf{J}_{n}^{j-1} \bar{u}_{b 2}^{j}
$$

where $t_{i j}^{(i)}$ is the element $i j$ in the matrix $\mathbf{T}^{(i)}$ in equation (16), and $\bar{u}_{b 2}^{j}$ is the displacement vector on the upper boundary for the $j$ th mode of the particular solutions in equation,(36). One should note that $\mathbf{J}_{n}^{j-1} \bar{u}_{b 2}^{j}=(1,0$, $-1)^{\mathrm{T}} \beta_{j}^{(1)},(0,1,0)^{\mathrm{T}} \beta_{j}^{(2)}$ or $(1,0,1)^{\mathrm{T}} \beta_{j}^{(3)}$.

After obtaining the stress vector on the upper boundary for each mode in equation (36), the displacement and stress fields given by the particular solutions in the interior domain can be expressed in terms of the displacement-stress vector at the upper boundary of the interior domain as shown in equations (16) and (19). The vector $\mathbf{J}^{-1} Y_{0}^{(i)}$ in equations (16) and (19) is the combination of the vectors $\mathbf{J}_{n}^{j-1} \bar{t}_{b 2}^{j}$ and $\mathbf{J}_{n}^{j-1} \bar{u}_{b 2}^{j}$ in equation (37) for the $j$ th mode of the particular solution. Therefore, the displacement and stress vectors on the upper boundary and the vertical interface, $S_{2}$ and $S_{3}$ in Figure 3(b), can be approximated by a finite number of lower modes as

$$
\begin{aligned}
& u_{\mathrm{p}, s 2}^{(\mathrm{i})}(r)=\mathbf{N}_{\mathrm{p}, \mathrm{s} 2}^{(\mathrm{i})}(r) \beta \\
& t_{\mathrm{p}, s 2}^{(\mathrm{i})}(r)=\mathbf{G}_{\mathrm{p}, s 2}^{(\mathrm{i})}(r) \beta
\end{aligned}
$$


and

$$
\begin{aligned}
u_{\mathrm{p}, s 3}^{(\mathrm{i})}(z) & =\mathbf{N}_{\mathbf{p}, \mathbf{s 3}}^{(\mathbf{i})}(z) \beta \\
t_{\mathbf{p}, \mathbf{s 3}}^{(\mathrm{i})}(z) & =\mathbf{G}_{\mathbf{p}, \mathbf{s} 3}^{(\mathrm{i})}(z) \beta
\end{aligned}
$$

where vector $\beta$ is the vector of participation factors in equation (36).

\subsection{Solutions for combined soil domain}

Once the homogeneous and the particular solutions have been found, the modal participation factors in equations (13), (23) and (23a) can be obtained by imposing the displacement and the stress continuity conditions of equations (4) and (5). Although the exact solutions for the interior and the exterior domains are given by infinite series, only a finite number of terms are needed to give a good approximation. The number of terms required depends on the desired accuracy. To enforce the conditions in equations (4) and (5), the weighted residual procedure is followed.

Considering the exterior domain as shown in Figure 3(a), the displacements and the tractions on the vertical interface $S_{1}+S_{3}$ can be found in equation (13). The prescribed displacements on $S_{1}+S_{3}$ can be obtained using the corresponding component in equation (1) for $S_{1}$ and equations (23a), (38a) for $S_{3}$. Then, the displacement continuity condition in equation (4) is imposed in the form of the variational principle $\int_{S_{1}+S_{3}} \delta t^{\mathrm{T}}(u-\bar{u}) \mathrm{d} s=0$ in which $\bar{u}$ is the prescribed displacement vector. This leads to

where

$$
K_{\mathrm{ee}} \alpha^{(\mathrm{e})}-K_{\mathrm{ei}} \alpha^{(\mathrm{i})}=V_{1} \beta+V_{b 1}
$$

$$
\begin{aligned}
K_{\mathrm{ee}} & =\int_{S_{1}+S_{3}} G^{(\mathrm{e})^{\mathrm{T}}}(z) N^{(\mathrm{e})}(z) \mathrm{d} z \\
K_{\mathrm{ei}} & =\int_{S_{3}} G^{(\mathrm{e})^{\mathrm{T}}}(z) N_{\mathrm{h}, 53}^{(\mathrm{i})}(z) \mathrm{d} z \\
V_{b 1} & =\int_{S_{1}} G^{(\mathrm{e})^{\mathrm{T}}}(z) \bar{u}_{b 1}(z) \mathrm{d} z \\
V_{1} & =\int_{S_{3}} G^{(\mathrm{e})^{\mathrm{T}}}(z) N_{\mathbf{p}, 53}^{(\mathrm{i})}(z) \mathrm{d} z
\end{aligned}
$$

For the interior domain shown in Figure 3(b), the displacements and the tractions on the interface $S_{3}$ can be obtained using equations (14), (23a) and (38a). Similarly, imposing the stress continuity condition in equation (5) for which the prescribed traction vector $t^{(e)}$ on $S_{3}$ can be found in equation (13), one can use the form of $\int_{S_{3}} \delta u^{\mathrm{T}}(t-\bar{t}) \mathrm{d} s=0$ with $\delta \beta=0$. This gives

$$
K_{i i} \alpha^{(i)}-K_{i e} \alpha^{(e)}=-V_{2} \beta
$$

where

$$
\begin{aligned}
K_{\mathrm{ii}} & =\int_{S_{3}} N_{\mathrm{h}, s 3}^{(\mathrm{i})^{\mathrm{T}}}(z) G_{\mathrm{h}, s 3}^{(\mathrm{i})}(z) \mathrm{d} z \\
K_{\mathrm{ie}} & =\int_{S_{3}} N_{\mathrm{h}, s 3}^{(\mathrm{i})^{\mathrm{T}}}(z) G^{(\mathrm{e})}(z) \mathrm{d} z=K_{\mathrm{ei}}^{\mathrm{T}} \\
V_{2} & =\int_{S_{3}} N_{\mathrm{h}, s 3}^{(\mathrm{i}) \mathrm{T}}(z) G_{\mathrm{p}, s 3}^{(\mathrm{i})}(z) \mathrm{d} s
\end{aligned}
$$

Combining equations (39) with (40), one obtains

$$
\left[\begin{array}{rr}
K_{\mathrm{ee}} & -K_{\mathrm{ei}} \\
-K_{\mathrm{ei}}^{\mathrm{T}} & K_{\mathrm{ii}}
\end{array}\right]\left[\begin{array}{l}
\alpha^{(\mathrm{e})} \\
\alpha^{(\mathrm{i})}
\end{array}\right]=\left[\begin{array}{r}
V_{1} \\
-V_{2}
\end{array}\right] \beta+\left[\begin{array}{l}
V_{b 1} \\
0
\end{array}\right]
$$


or

$$
K \alpha=V \beta+V_{b}
$$

Therefore, the unknown modal participation factors of the homogeneous solutions in the exterior and the interior domains can be calculated as follows:

$$
\alpha=K^{-1}\left(V \beta+V_{b}\right)
$$

Then the displacements and the stresses at any arbitary location in the soil domain or on the boundary can be calculated. Consequently the problem with arbitary prescribed displacements on the surface of the cylindrical cavity has been solved.

\section{ROOT SEARCHING SCHEME FOR TRANSCENDENTAL EQUATIONS}

Equations (8), (9), (17) and (18) have an infinite number of roots on the complex plane. However, only the roots in a specific region on the complex plane (Figure 4) are needed for engineering purposes without losing accuracy. The conventional complex root searching methods (e.g. Newton's and Muller's method ${ }^{11}$ ) often break down owing to the complexity of the transcendental functions and the lack of a good initial guess. Therefore, a newly developed root searching scheme is proposed.

Let $f(z)$ be the transcendental function

$$
f(z)=u(x, y)+\mathrm{i} v(x, y)
$$

where $z=x+\mathrm{i} y$ is a complex variable and $u(x, y)$ and $v(x, y)$ are the real and the imaginary parts of $f(z)$ respectively. The scheme is to find the approximate $z$ such that $f(z) \approx 0$.

To find all the approximate root $z$ 's, one needs to divide the region into the mesh shown in Figure 4 and calculate the function $f(z)$ at all the nodes. Assuming each grid in the mesh is small enough such that at most one root is located inside the grid, then one can check all the grids one by one as follows.

Figure 5 shows typical grid patterns. If any two function values among the function values at the four corners of the grid have different signs both in the real and the imaginary parts; i.e.

$$
u_{i} u_{j} \leqslant 0 \text { and } v_{i} v_{j} \leqslant 0, \quad 1 \leqslant i, j \leqslant 4 \text { and } i \neq j
$$

then one can use the Falsi or Muller methods ${ }^{11}$ to locate the points $z_{5}, z_{6}, z_{7}$ and $z_{8}$, as shown in Figures $5(\mathrm{a})$

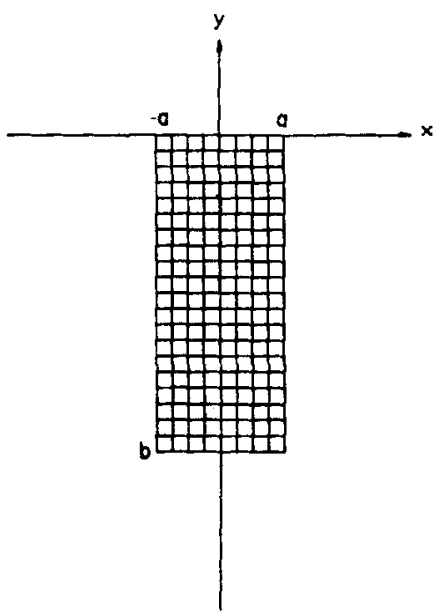

Figure 4. Mesh of the region $-a \leqslant x \leqslant a$ and $b \leqslant y \leqslant 0$ on complex plane 
(a)

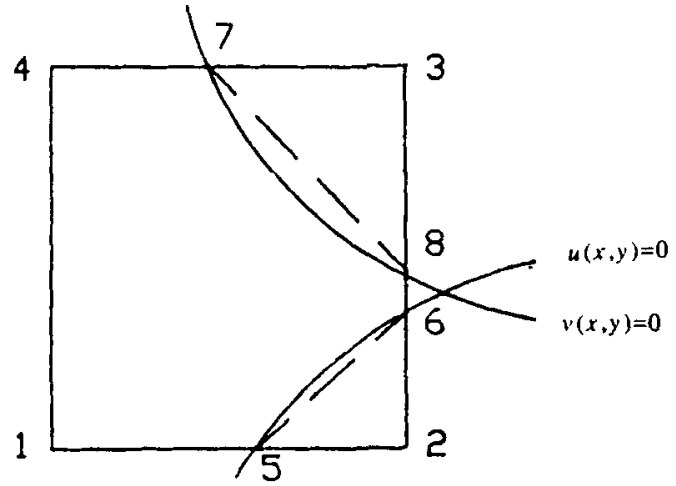

(c)

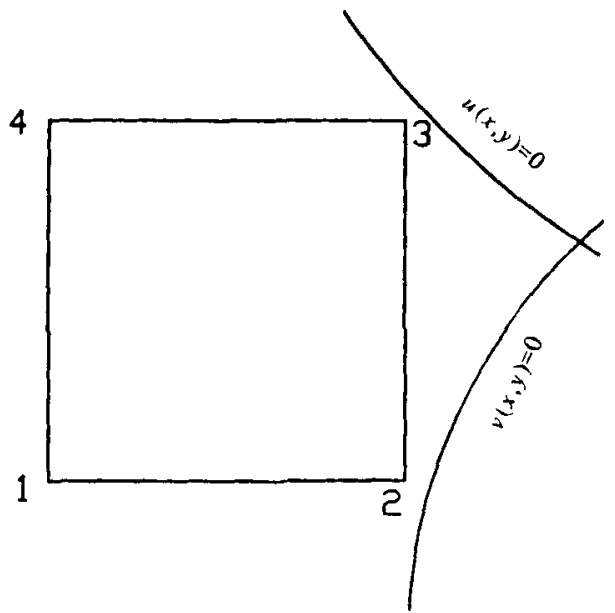

(b)

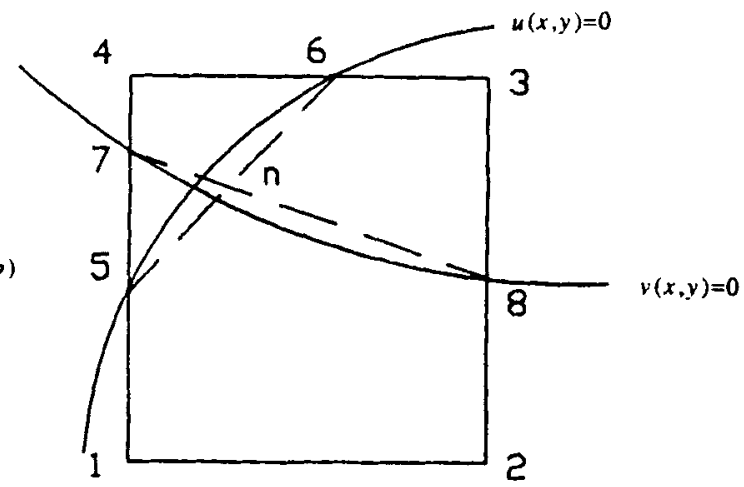

Figure 5. Grids without and with a root inside

and (b), at which

$$
\begin{array}{ll}
u\left(x_{5}, y_{5}\right)=0, & u\left(x_{6}, y_{6}\right)=0 \\
v\left(x_{7}, y_{7}\right)=0, & v\left(x_{8}, y_{8}\right)=0
\end{array}
$$

These four points can form two straight lines as shown in Figures 5(a) and (b). The line formed by $z_{5}$ and $z_{6}$ approximates $\operatorname{Ref}(f(z))=0$ in the grid and the equation for this line can be written as

$$
l_{\mathrm{r}}(x, y)=a_{1} x+b_{1} y+c_{1}=0
$$

where $a_{1}=y_{5}-y_{6}, b_{1}=x_{6}-x_{5}$ and $c_{1}=x_{5} y_{6}-y_{5} x_{6}$. Similarly, the line formed by $z_{7}$ and $z_{8}$ approximates $\operatorname{Im}(f(z))=0$ in the grid and the equation is

$$
l_{\mathbf{i}}(x, y)=a_{2} x+b_{2} y+c_{2}=0
$$

where $a_{2}=y_{7}-y_{8}, b_{2}=x_{8}-x_{7}$ and $c_{2}=x_{7} y_{8}-y_{7} x_{8}$.

To determine if there is a root in the grid, one can substitute $\left(x_{7}, y_{7}\right)$ and $\left(x_{8}, y_{8}\right)$ into $l_{\mathrm{r}}(x, y)$ in equation (46). If $l_{\mathrm{r}}\left(x_{7}, y_{7}\right) l_{\mathrm{r}}\left(x_{8}, y_{8}\right)>0$, then there is no root in the grid [Figure 5(a)]. On the other hand, $l_{\mathrm{r}}\left(x_{7}, y_{7}\right) l_{\mathrm{r}}\left(x_{8}, y_{8}\right) \leqslant 0$ means that there is a root in the grid [Figure 5(b)]. The approximate root $z_{n}$ is the intersection of the lines $l_{\mathrm{r}}(x, y)=0$ and $l_{\mathrm{i}}(x, y)=0$ in equations (46) and (47), and can be calculated using the following equations:

$$
\begin{gathered}
y_{n}=\frac{c_{1} a_{2}-c_{2} a_{1}}{b_{2} a_{1}-b_{1} a_{2}} \\
x_{n}=-\left(b_{1} y_{n}+c_{1}\right) / a_{1}, \quad \text { if } \quad a_{1} \geqslant a_{2}
\end{gathered}
$$


or

$$
x_{n}=-\left(b_{2} y_{n}+c_{2}\right) / a_{2}, \quad \text { if } \quad a_{2}>a_{1}
$$

After the approximate toot is found, one can improve the accuracy of the approximate root by the Newton method.

\section{NUMERICAL EXAMPLE}

The procedure described in the previous sections is demonstrated by a simple example of a rigid massless circular plate resting on a single layer stratum shown in Figure 6 and subjected to torsional and vertical excitations. Therefore, the prescribed boundary condition of equation (2) has only one term with $n=0$. Figure 6 also shows the dimensions and the soil properties of the total system. In order to avoid the singularity encountered in calculating the traction vector in equation (37) for some particular frequencies, material damping in the soil is necessary.

To find the impedances for the torsional and the vertical motions, both amplitudes of the harmonic displacement excitations are set equal to 1 . Then the impedances are the total reaction moment and force due to the corresponding stress under the plate.

For the case of torsional motion, the prescribed boundary condition on the top surface of the interior domain can be decomposed, using equation (36), as follows:

$$
\begin{aligned}
& \left\{\begin{array}{l}
\bar{u}_{r 2} \\
\bar{u}_{z 2} \\
\bar{u}_{\theta 2}
\end{array}\right\}=\left\{\begin{array}{l}
0 \\
0 \\
r
\end{array}\right\}=-\left\{\begin{array}{c}
1 \\
0 \\
-1
\end{array}\right\} \frac{r}{2}+\left\{\begin{array}{l}
1 \\
0 \\
1
\end{array}\right\} \frac{r}{2} \\
& =\sum_{i=0}^{\infty}-\left\{\begin{array}{c}
1 \\
0 \\
-1
\end{array}\right\} k_{i}^{(1)} \mathbf{J}_{1}\left(k_{i}^{(1)} r\right) \beta_{i}^{(1)}+\sum_{i=0}^{\infty}\left\{\begin{array}{l}
1 \\
0 \\
1
\end{array}\right\} k_{l}^{(3)} \mathbf{J}_{-1}\left(k_{l}^{(3)} r\right) \beta_{i}^{(3)} \quad 0 \leqslant r \leqslant \frac{1}{2} \\
& \begin{array}{l}
\text { Soil Properties } \\
\text { Hysteretic Damping Ratio } \xi=05 \\
\text { Shear Modulus } G=1+2 \xi i \\
\text { Poisson's Ratio } \mu=\frac{1}{3} \\
\text { Mass Density } \rho=1 \\
\text { Rigid Plate } \\
\end{array}
\end{aligned}
$$

Figure 6. Soil profile of example 
Since $k_{i}^{(1)}=k_{l}^{(3)}, \beta_{i}^{(1)}=-\beta_{l}^{(3)}$ and $\mathbf{J}_{1}\left(k_{i}^{(1)} r\right)=-\mathbf{J}_{-1}\left(k_{l}^{(3)} r\right)$ for $i=l$,

$$
\left\{\begin{array}{l}
\bar{u}_{r 2} \\
\bar{u}_{z 2} \\
\bar{u}_{\theta 2}
\end{array}\right\}=\sum_{i=0}^{\infty}-\left\{\begin{array}{c}
0 \\
0 \\
-1
\end{array}\right\} 2 k_{i}^{(1)} J_{1}\left(k_{i}^{(1)} r\right) \beta_{i}^{(1)} \quad 0 \leqslant r \leqslant \frac{1}{2}
$$

In the process of the decomposition, one should notice that matrix $\mathbf{J}_{n}$ in equation (24) is diagonal when $n=0$. In other words, vector $(0,0,-1)^{T}$ is an eigenvector of $J_{n}$ under the condition $n=0$. For the homogeneous solution, only the Love wave modes are involved. The wave number $k$ 's corresponding to the exterior and the interior domains are the roots of equations (9) and (18) respectively.

Similarly, for the case of vertical motion, the prescribed boundary condition can be decomposed as follows:

$$
\left\{\begin{array}{l}
\bar{u}_{r 2} \\
\bar{u}_{22} \\
\bar{u}_{\theta 2}
\end{array}\right\}=\left\{\begin{array}{l}
0 \\
1 \\
0
\end{array}\right\}=\sum_{j=0}^{\infty}\left\{\begin{array}{l}
0 \\
1 \\
0
\end{array}\right\} k_{j}^{(2)} \mathbf{J}_{0}\left(k_{j}^{(2)} r\right) \beta_{j}^{(2)} \quad 0 \leqslant r \leqslant \frac{1}{2}
$$

and only the Rayleigh wave modes are involved in the homogeneous solution. The wave number $k$ 's corresponding to the exterior and the interior domains are the roots of equations (8) and (17) respectively.

In this investigation, converged results can be obtained by using only the first seven modes of the particular solution of the interior domain [equation (50) or (51)]. For the homogeneous solutions, the roots of the transcendental equations [equations (8), (9), (17) and (18)] always come in pairs, i.e. both $k$ and $-k$ are roots. For the exterior domain, one should pick up the roots with a negative imaginary part, which satisfy the radiation condition of the far-field. Therefore, one needs only to search the roots of equations (8) and (9) on the lower half of the complex plane shown in Figure 4. For the interior domain, both $k$ and $-k$ roots give the same linear independent mode. Therefore, only the roots on the lower half of the complex plane are sought for equations (17) and (18).

While using the technique stated in Section 3 to find the roots of the transcendental equations, one has to decide the grid size first. After investigations, it is found that the roots, which are non-dimensional wave number $k h$ 's, with a large imaginary part, are quite far apart. For the cases with no material damping in the soil, only the roots on the real axis of the complex plane may be close to each other under very high frequency excitations. If the material damping is put in the soil, then these real roots deviate from the real axis. Therefore, for the cases with material damping in the soil, the strategy to determine the grid size near the real axis is to ignore the material damping in the soil and use the bisection method ${ }^{11}$ to locate the approximate real roots first. This suggests how far the roots are separated and one can use this information to determine the grid size near the real axis. For the roots with a large imaginary part, the grid size can be as large as $1.5 \times 1.5$.

In this example, the elements in matrices $\mathbf{K}$ and $\mathbf{V}$ in equation (41) are calculated by closed form integrations. The impedances for the torsional and the vertical modes are shown in Figures 7 and 8 respectively. In these figures, the non-dimensional frequency $\vec{a}_{0}=\omega a_{0} / c_{\mathrm{s}}, K_{\mathrm{T}}$ designates the torsional impedance and $K_{\mathrm{v}}$ represents the vertical impedance. The results obtained with 150 homogeneous modes in both the interior and the exterior domains are very close to that with 100 homogeneous modes. The four solid line curves in each figure represent the results obtained with 15,30,50 and 100 homogeneous modes in both domains respectively. From these results, one can see that the results converge in general. However, more homogeneous modes may be needed for higher frequency excitations in order to maintain the same accuracy as in the lower frequency range.

The dashed line curve in each figure is the result reported in Reference 8. Comparing both results with each other, one can notice some discrepancy. In Reference 8, the layer elements, which impose linear variation of the displacements in the elements in the vertical direction, are used to model the total system. This is similar to the finite element method. Therefore, the method of layer element approach theoretically gives the upper bound of the impedance. On the other hand, the method of analytic approach presented in this paper, which uses the weighted residual formulation to minimize the discrepancy at the vertical interface between the exterior and the interior domains, gives the lower bound of the impedance. This explains the small differences between the results obtained by the two methods. 

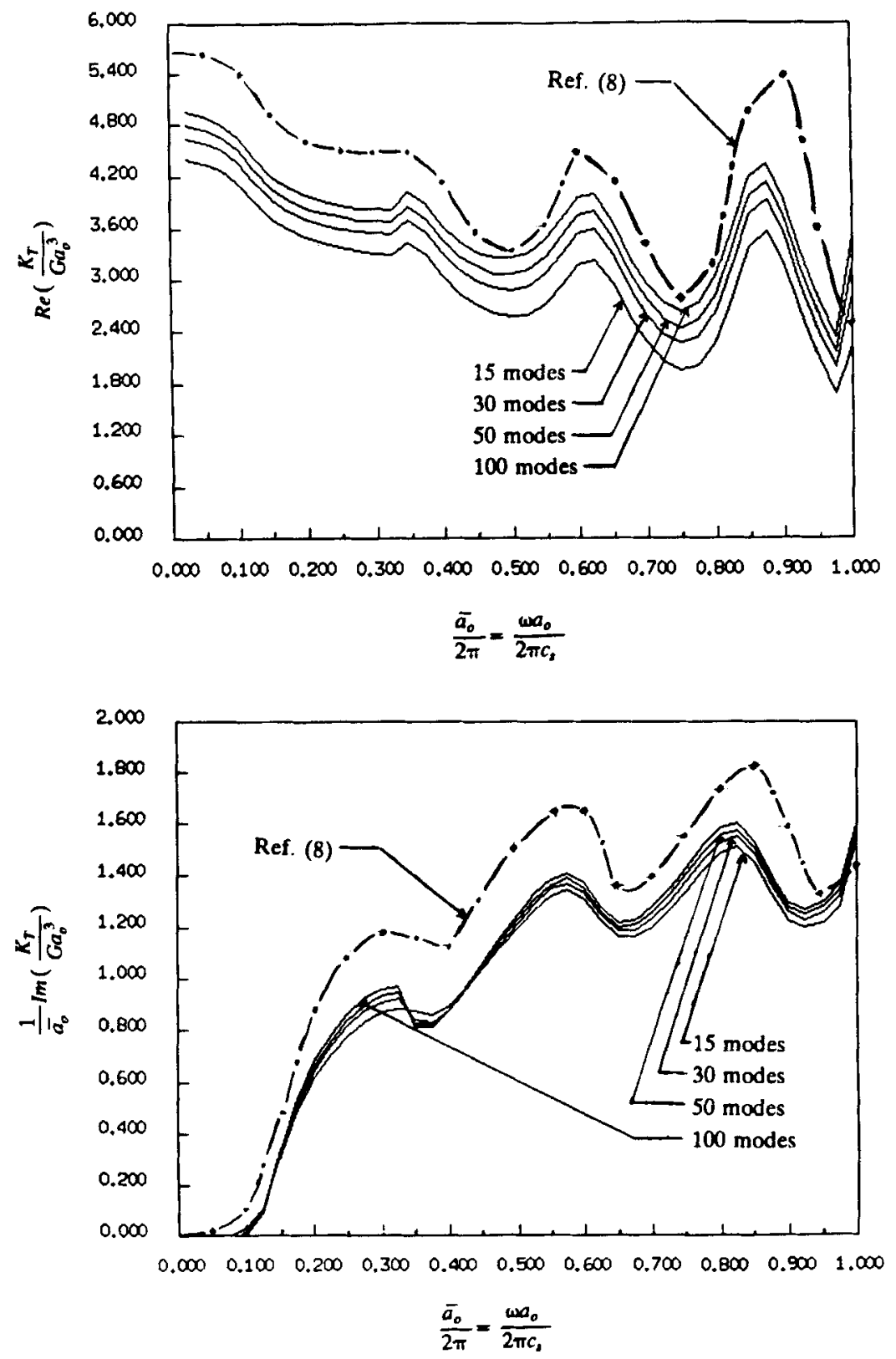

Figure 7. Torsional impedance

Furthermore, one can see that the method presented herein is more general than Tassoulas' procedure ${ }^{8}$ which can deal only with limited types of foundation (e.g. rigid circular or rigid ring foundation). The method presented has the same advantage as Tassoulas' procedure, requiring less storage in computations than Kausel's procedure. ${ }^{7.8}$ Therefore, the method presented is more suitable for the case of a deep soil stratum. Also, as pointed out previously, more modes may be needed for the higher frequency excitations. However, it is inconvenient to refine the layer element model in Tassoulas' or Kausel's procedure for the higher frequency excitations. The method presented can easily handle the problem by increasing the number of modes. Finally, another advantage of using this method is that the elements in matrices $\mathbf{K}$ and $\mathbf{V}$ in equation (41) can be calculated by closed form integrations which require less computational effort. 

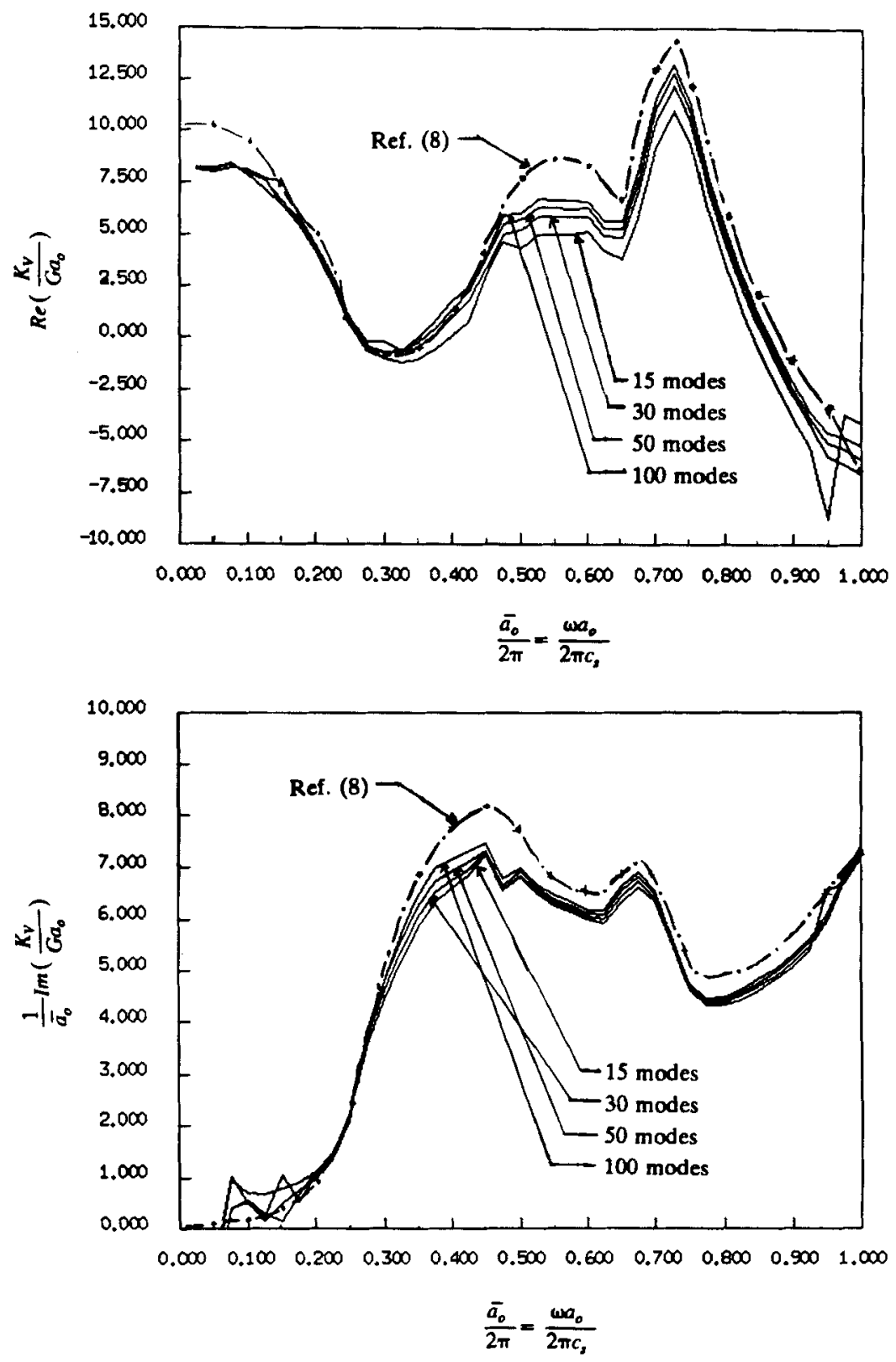

Figure 8. Vertical impedance

\section{DISCUSSION}

The method presented can also be applied to the problem with prescribed tractions, instead of prescribed displacements, acting on the surface of the cylindrical cavity. In such case, the boundary condition in equation (15) for the homogeneous solutions is replaced by a traction free condition and the prescribed tractions on the bottom of the cavity can be represented by three separate series of Bessel functions similar to equation (36). Procedures stated in the preceding sections are then followed to solve the problem.

The analytic solutions in the preceding sections can be easily combined with the finite element model of the structural domain. To do so, the displacement and the stress continuity conditions are imposed on the interface between the structural and the soil domains to generate the impedance matrix using the formulation 
of a weighted residual. In other words, the analytic solutions can be used to replace the Green's functions as the fundamental modes in the boundary element method provided the bedrock under the layered soil stratum can be assumed rigid.

To perform the soil-structure interaction analysis, one is usually interested in the displacement and the stress fields in the soil vicinity near the foundation level of the structure. For the case with material damping in the soil medium, the stress wave attenuates along the travelling path. Therefore, if the material damping in the soil medium is significant, and the rigid lower boundary is at a distance sufficiently far away so that the reflected waves from the rigid boundary have little effect on the results, then the analytic solutions reported in this paper can be an approximation for a layered half-space medium. In the approximation, the depth of the lowered rigid boundary will depend upon the magnitude of the material damping in the soil, the dimensions of the foundation, the depth of the embedment of the foundation and the excitation frequency. The relationship between these parameters needs further study.

\section{ACKNOWLEDGEMENTS}

The author wishes to thank Dr David Lau who reviewed the manuscript and gave suggestions, and $\mathrm{Mr}$ Xiaoxuan Qi who helped draw the figures.

\section{APPENDIX}

The transfer matrice $\mathbf{a}_{j}$ 's in equations (6) and (16) can be expressed as follows: ${ }^{9}$

$$
a_{j}=\left[\begin{array}{cc}
a_{1} & 0 \\
0 & a_{2}
\end{array}\right]
$$

in which

$$
\mathbf{a}_{1}=\left[\begin{array}{cc}
\frac{2 k^{2}}{k_{\beta}^{2}}\left(\mathrm{CH}-\mathrm{CH}^{\prime}\right)+\mathrm{CH}^{\prime} & -\frac{k}{k_{\beta}^{2}}\left(\left(2 k^{2}-k_{\beta}^{2}\right) \frac{\mathrm{SH}}{v} 2 v^{\prime} \mathrm{SH}^{\prime}\right) \\
\frac{k}{k_{\beta}^{2}}\left(2 v \mathrm{SH}-\left(2 k^{2}-k_{\beta}^{2}\right) \frac{\mathrm{SH}^{\prime}}{v^{\prime}}\right) & \mathrm{CH}-\frac{2 k^{2}}{k_{\beta}^{2}}\left(\mathrm{CH}-\mathrm{CH}^{\prime}\right) \\
G\left(\frac{4 k^{2}}{k_{\beta}^{2}} v \mathrm{SH}-\frac{\left(2 k^{2}-k_{\beta}^{2}\right)^{2}}{k_{\beta}^{2}} \frac{\mathrm{SH}^{\prime}}{v^{\prime}}\right. & -\frac{2 k G}{k_{\beta}^{2}}\left(2 k^{2}-k_{\beta}^{2}\right)\left(\mathrm{CH}-\mathrm{CH}^{\prime}\right) \\
\frac{2 k G}{k_{\beta}^{2}}\left(2 k^{2}-k_{\beta}^{2}\right)\left(\mathrm{CH}-\mathrm{CH}^{\prime}\right) & G\left(-\frac{\left(2 k^{2}-k_{\beta}^{2}\right)^{2}}{k_{\beta}^{2}} \frac{\mathrm{SH}}{v}+\frac{4 k^{2}}{k_{\beta}^{2}} v^{\prime} \mathrm{SH}^{\prime}\right) \\
\frac{-1}{G k_{\beta}^{2}}\left(v^{\prime} \mathrm{SH}-k^{2} \frac{\mathrm{SH}}{v}\right) & -\frac{k}{G k_{\beta}^{2}}\left(\mathrm{CH}-\mathrm{CH}^{\prime}\right) \\
\frac{k}{G k_{\beta}^{2}}\left(\mathrm{CH}-\mathrm{CH}^{\prime}\right) & \frac{-1}{G k_{\beta}^{2}}\left(v \mathrm{SH}-k^{2} \frac{\mathrm{SH}^{\prime}}{v^{\prime}}\right) \\
\frac{2 k^{2}}{k_{\beta}^{2}}\left(\mathrm{CH}-\mathrm{CH}^{\prime}\right)+\mathrm{CH}^{\prime} & -\frac{k}{k_{\beta}^{2}}\left(2 v \mathrm{SH}-\left(2 k^{2}-k_{\beta}^{2}\right) \frac{\mathrm{SH}^{\prime}}{v^{\prime}}\right) \\
\frac{\left.\left(2 k^{2}-k_{\beta}^{2}\right) \frac{\mathrm{SH}}{v}-2 v^{\prime} \mathrm{SH}^{\prime}\right)}{\mathrm{CH}-\frac{2 k^{2}}{k_{\beta}^{2}}\left(\mathrm{CH}-\mathrm{CH}^{\prime}\right)}
\end{array}\right]
$$


and

$$
\mathbf{a}_{2}=\left[\begin{array}{cc}
\mathrm{CH}^{\prime} & \frac{\mathbf{S} \mathbf{H}^{\prime}}{G v^{\prime}} \\
G v^{\prime} \mathrm{SH}^{\prime} & \mathbf{C H ^ { \prime }}
\end{array}\right]
$$

$\mathrm{CH}=\cosh v d_{j}, \mathrm{CH}^{\prime}=\cosh v^{\prime} d_{j}, \mathrm{SH}=\sinh v d_{j}, \mathrm{SH}^{\prime}=\sinh v^{\prime} d_{j}, k_{\beta}=\sqrt{ }\left(\omega^{2} / c_{\mathrm{s}}^{2}\right), G$ is the shear modulus, and $v$ and $v^{\prime}$ are given in equation (24).

The Hankel function matrix $\mathbf{H}$ in equation (6) is

$$
\mathbf{H}=\left[\begin{array}{cccccc}
H_{n}^{\prime} & 0 & 0 & 0 & \frac{n}{r} H_{n} & 0 \\
0 & k H_{n} & 0 & 0 & 0 & 0 \\
0 & 0 & H_{n}^{\prime} & 0 & 0 & \frac{n}{r} H_{n} \\
0 & 0 & 0 & k H_{n} & 0 & 0 \\
\frac{n}{r} H_{n} & 0 & 0 & 0 & H_{n}^{\prime} & 0 \\
0 & 0 & \frac{n}{r} H_{n} & 0 & 0 & H_{n}^{\prime}
\end{array}\right]
$$

in which $H_{n}=H_{n}^{(2)}(k r)$ is the second kind of Hankel function of order $n$ and $H_{n}^{\prime}=\mathrm{d} H_{n} / \mathrm{d} r$. The Bessel function matrix $\mathbf{J}$ in equation (16) has the same form as the matrix $\mathbf{H}$ except that $H_{n}$ and $H_{n}^{\prime}$ in $\mathbf{H}$ are replaced by $J_{n}$ and $J_{n}^{\prime}$ respectively, $J_{n}$ is the first kind of Bessel function of order $n$ and $J_{n}^{\prime}=\mathrm{d} J_{n} / \mathrm{d} r$.

The matrices $\mathbf{F}_{1} e\left(z-h_{j-1}\right) \mathbf{E}_{j}^{-1}$ and $\mathbf{F}_{2} e\left(z-h_{j-1}\right) \mathbf{E}_{j}^{-1}$ in equations (10) and (19) can be expressed as follows:

$$
\begin{aligned}
& \mathbf{F}_{1} e\left(z-h_{j-1}\right) \mathbf{E}_{j}^{-1}=\left[\begin{array}{cc}
\frac{G}{k_{\beta}^{2}}\left(4 k^{2} v \mathrm{SH}-\left(2 k^{2}-k_{\beta}^{2}\right)^{2} \frac{\mathrm{SH}^{\prime}}{v^{\prime}}\right) & -\frac{2 k G}{k_{\beta}^{2}}\left(2 k^{2}-k_{\beta}^{2}\right)\left(\mathrm{CH}-\mathrm{CH}^{\prime}\right) \\
\frac{2 k G}{k_{\beta}^{2}}\left(-\left(2 v^{2}+k_{\beta}^{2}\right) \mathrm{CH}+\left(2 k^{2}-k_{\beta}^{2}\right) \mathrm{CH}^{\prime}\right) & \frac{G}{k_{\beta}^{2}}\left(\left(2 k^{2}-k_{\beta}^{2}\right)\left(2 v^{2}+k_{\beta}^{2}\right) \frac{\mathrm{SH}}{v}-4 k^{2} v^{\prime} \mathrm{SH}^{\prime}\right) \\
0 & 0
\end{array}\right. \\
& \frac{2 k^{2}}{k_{\beta}^{2}}\left(\mathrm{CH}-\mathrm{CH}^{\prime}\right)+\mathrm{CH}^{\prime} \quad \frac{k}{k_{\beta}^{2}}\left(\left(2 k^{2}-k_{\beta}^{2}\right) \frac{\mathrm{SH}^{\prime}}{v^{\prime}}-2 v \mathrm{SH}\right) 0 \quad 0 \\
& \frac{k}{k_{\beta}^{2}}\left(2 v^{\prime} \mathrm{SH}^{\prime}-\left(2 v^{2}+k_{\beta}^{2}\right) \frac{\mathrm{SH}}{v}\right) \frac{1}{k_{\beta}^{2}}\left(\left(2 v^{2}+k_{\beta}^{2}\right) \mathrm{CH}-2 k^{2} \mathrm{CH}^{\prime}\right) \quad 0 \quad 0 \\
& 0 \quad \begin{array}{lll}
0 & G v^{\prime} \mathrm{SH}^{\prime} & \mathrm{CH}^{\prime}
\end{array}
\end{aligned}
$$

and

$$
\begin{aligned}
& \mathbf{F}_{2} e\left(z-h_{j-1}\right) \mathbf{E}_{j}^{-1}=\left[\begin{array}{cc}
2 G\left(\frac{2 k^{2}}{k_{\beta}^{2}}\left(\mathrm{CH}-\mathrm{CH}^{\prime}\right)+\mathrm{CH}^{\prime}\right) & \frac{2 k G}{k_{\beta}^{2}}\left(2 v^{\prime} \mathrm{SH}^{\prime}-\left(2 k^{2}-k_{\beta}^{2}\right) \frac{\mathrm{SH}}{v}\right) \\
0 & 0
\end{array}\right. \\
& \left.\begin{array}{cccc}
\frac{2}{k_{\beta}^{2}}\left(k^{2} \frac{\mathrm{SH}}{v}-v^{\prime} \mathrm{SH}^{\prime}\right) & -\frac{2 k}{k_{\beta}^{2}}\left(\mathrm{CH}-\mathrm{CH}^{\prime}\right) & 0 & 0 \\
0 & 0 & 2 G \mathrm{CH}^{\prime} & 2 \frac{\mathrm{SH}^{\prime}}{v^{\prime}}
\end{array}\right]
\end{aligned}
$$


in which $\mathrm{CH}=\cosh v\left(z-h_{j-1}\right), \mathrm{CH}^{\prime}=\cosh v^{\prime}\left(z-h_{j-1}\right), \mathrm{SH}=\sinh v\left(z-h_{j-1}\right)$ and $\mathrm{SH}^{\prime}=\sinh v^{\prime}\left(z-h_{j-1}\right)$.

The matrices $H_{1}$ and $H_{2}$ in equation (10) can be expressed as follows:

$$
\mathbf{H}_{1}=\left[\begin{array}{ccc}
0 & k H_{n} & 0 \\
H_{n}^{\prime} & 0 & \frac{n}{r} H_{n} \\
0 & 0 & 0
\end{array}\right]
$$

and

$$
\mathbf{H}_{2}=\left[\begin{array}{cc}
\left(-\frac{H_{n}^{\prime}}{r}+\frac{n^{2}}{r^{2}} H_{n}\right) & \left(\frac{n}{r} H_{n}^{\prime}-\frac{n}{r^{2}} H_{n}\right) \\
0 & 0 \\
\left(\frac{n}{r} H_{n}^{\prime}-\frac{n}{r^{2}} H_{n}\right) & \left(-\frac{H_{n}^{\prime}}{r}+\left(\frac{n^{2}}{r^{2}}-\frac{k^{2}}{2}\right) H_{n}\right)
\end{array}\right]
$$

The matrices $\mathbf{J}_{1}$ and $\mathbf{J}_{2}$ in equation (19) have the same forms as the matrices $\mathbf{H}_{1}$ and $\mathbf{H}_{2}$ except that $H_{n}$ and $H_{n}^{\prime}$ are replaced by $J_{n}$ and $J_{n}^{\prime}$ respectively.

\section{REFERENCES}

1. C.-H. Chen and J. Penzien, 'Seismic modelling of deep foundation', Report No. UCB/EERC-84/19, Earthquake Engineering Research Center, University of California, Berkeley, CA, 1984.

2. S. Gupta, T.-W. Lin, J. Penzien and C.-S. Yeh, 'Hybrid-modelling of soil-structure interaction', Report No. UCB/EERC-80/09, Earthquake Engineering Research Center, University of California, Berkeley, CA, 1980.

3. K. Muto, T. Murata, M. Takahashi and M. Kamata, 'Analytical study on soil-structure interaction of the embedded structure by . boundary element method', Muto Report 83-9-6, Tokyo, Japan, 1983.

4. J. P. Wolf and G. R. Darbre, 'Dynamic stiffness matrix of soil by boundary element method: Conceptual aspects', Earthquake eng. struct. dyn. 12, 385-400 (1984).

5. R. J. Apsel, 'Dynamic Green's functions for layered media and applications to boundary-value problems', Ph.D. Dissertation, UC San Diego, CA, 1979.

6. G. Waas, 'Linear two-dimensional analysis of soil dynamic problems in semi-infinite layered media', Ph.D. Dissertation, UCB, 1972.

7. E. Kausel, 'Forced vibrations of circular foundations on layered media', R74-11, Department of Civil Engineering, MIT, 1974.

8. J. L. Tassoulas, 'Elements for the numerical analysis of wave motion in layered media', $R 81-2$, Department of Civil Engineering, MIT, 1981.

9. T.-J. Tzong and J. Penzien, 'Hybrid modelling of soil-structure interaction in layered media', Report No. UCB/EERC-83/22, Earthquake Engineering Research Center, University of California, Berkeley, CA, 1983.

10. K. Sezawa, 'Further studies on Rayleigh waves having some azimuthal distribution', Bull. earthquake res. inst. Tokyo Univ. 6, 1-18 (1929).

11. S. D. Conte and C. de Boor, Elementary Numerical Analysis, McGraw-Hill, New York, 1980, pp. 72-127. 